\title{
AN ALGEBRAIC PROOF OF THE BORSUK-ULAM THEOREM FOR POLYNOMIAL MAPPINGS
}

\author{
MANFRED KNEBUSCH ${ }^{1}$
}

\begin{abstract}
An algebraic proof is given for the following theorem: Every system of $n$ odd polynomials in $n+1$ variables over a real closed field $R$ has a common zero on the unit sphere $S^{n}(R) \subset R^{n+1}$.
\end{abstract}

In [1] Dai, Lam, and Peng prove the remarkable theorem that for any natural number $n$ the quotient

$$
A_{n}(\mathbf{R}):=\mathbf{R}\left[X_{1}, \ldots, X_{n}\right] /\left(1+X_{1}^{2}+\cdots+X_{n}^{2}\right)
$$

of the polynomial ring $\mathbf{R}\left[X_{1}, \ldots, X_{n}\right]$ has level $n$, i.e. -1 cannot be written as a sum of less than $n$ squares in this ring. Besides an ingenious algebraic manipulation, their proof uses the following theorem.

THEOREM 1. Let $q_{1}, \ldots, q_{n-1}$ be polynomials in $\mathbf{R}\left[X_{1}, \ldots, X_{n}\right]$ which are odd, i.e. $q_{j}\left(-X_{1}, \ldots,-X_{n}\right)=-q_{j}\left(X_{1}, \ldots, X_{n}\right)$ for $1 \leqslant j \leqslant n-1$. Then these polynomials have a common zero on the unit sphere $S^{n-1} \subset \mathbf{R}^{n}$.

This theorem is equivalent to

THEOREM 1a. Let $g_{1}, \ldots, g_{n-1}$ be polynomials in $\mathbf{R}\left[X_{1}, \ldots, X_{n}\right]$. Then there exists some point $a$ in $S^{n-1}$ with $g_{j}(-a)=g_{j}(a)$ for $1<j<n-1$.

Hint. Write every $g_{j}$ as a sum of an even and an odd polynomial.

Theorem la is a special case of the well-known Borsuk-Ulam theorem, which states the same for continuous functions $g_{j}$ on $S^{n-1}$ instead of polynomials. It is this highly nontrivial fact from algebraic topology that Dai, Lam, and Peng refer to in their proof that $A_{n}(\mathbf{R})$ has level $n$. In order to eliminate the topological argument, I shall give in this note a purely algebraic proof of Theorem 1.

Lam has shown me the proofs of many other algebraic results on sums of squares and higher powers, partially announced in [1], which he and his collaborators gained by topological methods. Up to now I know of no way to prove any of these results algebraically.

As an algebraic proof should do, our proof of Theorem 1 will work equally well for any real closed base field $R$ instead of $\mathbf{R}$. Then the arguments of Dai, Lam, and Peng yield, without use of Tarski's principle or similar considerations, that every ring $A_{n}(R)$ has level $n$.

Received by the editors December 22, 1980 and, in revised form, March 10, 1981.

1980 Mathematics Subject Classification. Primary 14G30; Secondary 55M20.

'Supported by the DFG. 
Thus we are now given odd polynomials $q_{1}, \ldots, q_{n-1}$ in $R\left[X_{1}, \ldots, X_{n}\right]$ of total degrees $d_{1}, \ldots, d_{n-1}$ (odd numbers), and we have to look for a common zero of these polynomials on the sphere

$$
S^{n-1}(R):=\left\{\left(x_{1}, \ldots, x_{n}\right) \in R^{n} \mid x_{1}^{2}+\cdots+x_{n}^{2}=1\right\} .
$$

This needs some preparations. Let $C$ be the algebraic closure $R(\sqrt{-1})$ of $R$. For any odd number $d$ we denote by $F(d)$ the vector space over $C$ consisting of all forms $f$ in $C\left[X_{0}, \ldots, X_{n}\right]$ of degree $d$ which only contain monomials $X_{0}^{\alpha_{0}} X_{1}^{\alpha_{1}} \ldots X_{n}^{\alpha_{n}}$ with $\alpha_{1}+\cdots+\alpha_{n}$ odd. We fix odd numbers $d_{1}, \ldots, d_{n-1}$ and introduce the vector space

$$
F:=F\left(d_{1}\right) \times \cdots \times F\left(d_{n-1}\right) .
$$

Notice that the homogenizations $q_{1}^{*}, \ldots, q_{n-1}^{*}$ of the polynomials $q_{1}, \ldots, q_{n-1}$ above yield a point $\left(q_{1}^{*}, \ldots, q_{n-1}^{*}\right)$ in this space $F$. Let $V$ denote the quadric $x_{0}^{2}-x_{1}^{2}-\cdots-x_{n}^{2}=0$ in the projective space $P^{n}(C)$. The sphere $S^{n-1}(R)$ will be identified with the set $V(R)$ of real points of $V$ in the usual way. We introduce the closed subvariety $X$ of $F \times V$, consisting of all points $\left(f_{1}, \ldots, f_{n-1}\right.$; $\left.x_{0}: x_{1}: \cdots: x_{n}\right)$ with $f_{j}\left(x_{0}, x_{1}, \ldots, x_{n}\right)=0$ for $1<j<n-1$, and we finally introduce the natural projection $\pi: X \rightarrow F$. Notice that $\pi$ is a projective morphism defined over $R$. For every point $\left(f_{1}, \ldots, f_{n-1}\right) \in F$ the fibre $\pi$ reads as follows:

$$
\pi^{-1}\left(f_{1}, \ldots, f_{n-1}\right)=\left\{\left(f_{1}, \ldots, f_{n-1}\right)\right\} \times Z_{V}\left(f_{1}, \ldots, f_{n-1}\right)
$$

with $Z_{V}\left(f_{1}, \ldots, f_{n-1}\right)$ the set of common zeros of the forms $f_{1}, \ldots, f_{n-1}$ in $V$.

We compute this fibre for some special points of $F$. We choose finite sets $S_{1}, \ldots, S_{n-1}$ in $R$ consisting of $d_{1}, d_{2}, \ldots, d_{n-1}$ elements respectively. We consider the point

$$
p\left(S_{1}, \ldots, S_{n-1}\right):=\left(\prod_{a \in S_{1}}\left(X_{1}-a X_{n}\right), \ldots, \prod_{a \in S_{n-1}}\left(X_{n-1}-a X_{n}\right)\right)
$$

of $F$. (Notice that the variable $X_{0}$ is absent here!) Clearly

$$
\begin{aligned}
& \pi^{-1}\left(p\left(S_{1}, \ldots, S_{n-1}\right)\right)=\left\{p\left(S_{1}, \ldots, S_{n-1}\right)\right\} \\
& \quad \times\left\{\left( \pm \sqrt{1+a_{1}^{2}+\cdots+a_{n-1}^{2}}: a_{1}: \cdots: a_{n-1}: 1\right) \mid a_{1} \in S_{1}, \ldots, a_{n-1} \in S_{n-1}\right\} .
\end{aligned}
$$

Thus this fibre contains precisely $2 d_{1} \cdots d_{n-1}$ points. They happen to be all real.

Let $\tilde{U}$ denote the set of all points $p$ of $F$ with finite fibre $\pi^{-1}(p)$. By general principles from algebraic geometry $\tilde{U}$ is Zariski open in $F$, and in our case $\tilde{U}$ is defined over $R$ and nonempty. The restriction $\pi^{-1}(\tilde{U}) \rightarrow \tilde{U}$ of $\pi$ is a projective morphism with finite fibres, hence a finite morphism. By Bezout's theorem every fibre $\pi^{-1}(p)$ with $p \in \tilde{U}$ contains at most $2 d_{1} \cdots d_{n-1}$ elements. We have seen above that there exist fibres with precisely this cardinality. It is now clear from the general theory of finite morphisms that the set

$$
U:=\left\{p \in \tilde{U} \mid \operatorname{Card}\left(\pi^{-1}(p)\right)=2 d_{1} \cdots d_{n-1}\right\}
$$

is Zariski open in $\tilde{U}$ and defined over $R$. (It is the complement of the "discriminant hypersurface" in $\tilde{U}$.) 
We now look at the closed subvariety $Y:=X \cap(F \times W)$ of $X$ with $W:=\left\{\left(0: x_{1}: \cdots: x_{n}\right) \mid x_{1}^{2}+\cdots+x_{n}^{2}=0\right\}$. Since $\pi$ is projective and defined over $R$ the image $\pi(Y)$ is a closed subvariety of $F$ defined over $R$. ( $\pi(Y)$ may be reducible.) Clearly no one of the points $p\left(S_{1}, \ldots, S_{n-1}\right)$ from above lies in $\pi(Y)$. Thus certainly $\pi(Y) \neq F$.

We denote by $F_{1}$ the Zariski open subset of $F$, defined over $R$, which consists of all points $\left(f_{1}, \ldots, f_{n-1}\right)$ with $f_{j}\left(0, X_{1}, \ldots, X_{n}\right) \neq 0$ for $1<j<n-1$. This space $F_{1}$ will be identified via dehomogenization with the space of all systems $\left(q_{1}, \ldots, q_{n-1}\right)$ of odd polynomials $q_{1}, \ldots, q_{n-1}$ in $C\left[X_{1}, \ldots, X_{n}\right]$ of total degrees $d_{1}, \ldots, d_{n-1}$ respectively.

We introduce the Zariski open subset $U_{1}:=F_{1} \cap U \cap(F \backslash \psi(Y))$ of $F_{1}$ which is defined over $R$. Since $F$ is an irreducible variety, in fact a vector space, $U_{1}$ is certainly nonempty. For every point $p=\left(q_{1}, \ldots, q_{n-1}\right)$ of $U_{1}$ all common zeros of the homogenizations $q_{1}^{*}, \ldots, q_{n-1}^{*}$ on $V$ have first coordinate $x_{0} \neq 0$, since $p \notin$ $\psi(Y)$. Thus they are the points $\left(1: a_{1}: \cdots: a_{n}\right)$ with $q_{j}\left(a_{1}, \ldots, a_{n}\right)=0$ for $1<j<$ $n-1$ and $a_{1}^{2}+\cdots+a_{n}^{2}=1$. We know from above that for every $p \in U_{1}$ there exist precisely $2 d_{1} \cdots d_{n-1}$ such points. Thus we have arrived at the following lemma.

LEMMA. Let $d_{1}, \ldots, d_{n-1}$ be fixed odd numbers and let $F_{1}$ denote the space of all systems $\left(q_{1}, \ldots, q_{n-1}\right)$ of odd polynomials $q_{j}$ in $C\left[X_{1}, \ldots, X_{n}\right]$ of total degrees $d_{j}$ $(1<j<n-1)$. Then there exists a nonempty Zariski-open subset $U_{1}$ of $F_{1}$, defined over $R$, such that every system $\left(q_{1}, \ldots, q_{n-1}\right) \in U_{1}$ has precisely $2 d_{1} \cdots d_{n-1}$ common zeros on the "complex sphere"

$$
S^{n-1}(C):=\left\{\left(x_{1}, \ldots, x_{n}\right) \in C^{n} \mid x_{1}^{2}+\cdots x_{n}^{2}=1\right\} .
$$

We now enter the proof of Theorem 1 . We look at the set $F_{1}(R)$ of all systems $\left(q_{1}, \ldots, q_{n-1}\right)$ of odd polynomials $p_{j}$ in $R\left[X_{1}, \ldots, X_{n}\right]$ of degrees $d_{j}$. Identifying every $q_{j}$ with the tuple of its coefficients we regard $F_{1}(R)$ as a Zariski open subset of some vector space $R^{N}$. We work with the strong topology of $R^{N}$, which stems from the ordering of the field $R$. The set $F_{1}(R)$ is open in $R^{N}$, also in this topology.

Suppose there exists some system $\left(q_{1}^{0}, \ldots, q_{n-1}^{0}\right) \in F_{1}(R)$ which has no common zero on the real sphere $S^{n-1}(R)$. The polynomial $\left(q_{1}^{0}\right)^{2}+\cdots+\left(q_{n-1}^{0}\right)^{2}$ attains on $S^{n-1}(R)$ its minimum (cf. [2, §9] for an algebraic proof in the spirit of this paper). Thus $q_{1}^{0}(x)^{2}+\cdots+q_{n-1}^{0}(x)^{2}>\varepsilon$ for some $\varepsilon>0$ in $R$ and every $x \in S^{n-1}$. It is now clear that there exists a euclidean open ball $B$ in $R^{N}$ with center $\left(q_{1}^{0}, \ldots, q_{n-1}^{0}\right)$ and contained in $F_{1}(R)$ such that

$$
q_{1}(x)^{2}+\cdots+q_{n-1}(x)^{2}>0
$$

for every $\left(q_{1}, \ldots, q_{n-1}\right) \in B$ and every $x \in S^{n-1}(R)$. This means that no system $\left(q_{1}, \ldots, q_{n-1}\right) \in B$ has a common zero on $S^{n-1}(R)$. The semialgebraic set $B$ has dimension $N$, but $F^{1}(R) \backslash U_{1}(R)$, with $U_{1}$ from the lemma, has dimension at most $N-1$ (cf. [2, §8] for the dimension theory of semialgebraic sets over $R$ ). Thus $B \cap U_{1}(R) \neq \varnothing$. We now choose some system $\left(q_{1}, \ldots, q_{n-1}\right) \in B \cap U_{1}(R)$ and 
consider the set

$$
Z:=\left\{a \in S^{n-1}(C) \mid q_{1}(a)=\cdots=q_{n-1}(a)=0\right\} .
$$

By the lemma above $Z$ has the cardinality $|Z|=2 d_{1} \cdots d_{n-1}$. Since $\left(q_{1}, \ldots, q_{n-1}\right) \in B$ the intersection $Z \cap S_{n-1}(R)$ is empty, i.e. $Z$ contains no real points.

For every point $a$ of $Z$ the complex conjugate point $\bar{a}$ lies again in $Z$, since the polynomials $q_{j}$ have real coefficients. Also the antipodal point $-a$ lies in $Z$, since the $q_{j}$ are odd. Thus the Kleinian four-group $G=\{1, \sigma, \tau, \sigma \tau\}$ acts on $Z$ via $\sigma(a)=\bar{a}, \tau(a)=-a$. I claim that the action of $G$ is free of fixed points. Of course $\tau(a) \neq a$ for every $a \in Z$. Also $\sigma(a) \neq a$ for every $a \in Z$, since $Z$ does not contain real points. Assume that $\sigma \tau(a)=a$ for some $a \in Z$. This means $\bar{a}=-a$, i.e. that the coordinates $a_{1}, \ldots, a_{n}$ of $a$ are all purely imaginary. But this is impossible since $a_{1}^{2}+\cdots+a_{n}^{2}=1$. Thus the action of $F$ is indeed free. This implies that 4 divides $|Z|$, which is the desired contradiction, since $|Z|=2 d_{1} \cdots d_{n-1}$ is only divisible by 2 . Theorem 1 is proved.

ACKNOWLEDGEMENTS. I thank the University of California and in particular T. Y. Lam for a pleasant stay at Berkeley, during which this paper emerged, and the Deutsche Forschungsgemeinschaft for the financial support which made this possible.

ADDENDUM (FEBRUARY 1981). Leaving the cadre of pure algebra it is of course possible to deduce from Theorem 1 or Theorem la immediately the full BorsukUlam theorem. One simply approximates the given continuous functions on the sphere by polynomials by use of the Stone-Weierstrass theorem, as has been pointed out to me by several colleagues. The situation here is slightly unsatisfactory if the base field $\mathbf{R}$ is replaced by a nonarchimedian real closed base field $R$. Then the Borsuk-Ulam theorem can still be established for continuous semialgebraic functions instead of polynomials by copying the classical proof, replacing the singular homology by "semialgebraic homology" (Thesis of Hans Delfs, Regensburg 1980). But the Stone-Weierstrass theorem is known to be wrong over a nonarchimedian real closed base field. Thus our algebraic proof does not yield the full semialgebraic Borsuk-Ulam theorem in such a simple way.

\section{REFERENCES}

1. Z. D. Dai, T. Y. Lam and C. K. Peng, Levels in algebra and topology, Bull. Amer. Math. Soc. (N.S.) 3 (1980), 845-848.

2. H. Delfs and M. Knebusch, Semialgebraic topology. II. Basic theory of semialgebraic spaces, Math. Z. (to appear).

Fakultät für Mathematik der Universitüt, D-8400 Regensburg, Federal Repbulic of GERMANY 